\title{
Frontières
}

\section{Une carrière en marge de l'autre}

\section{Entretien avec un criminologue au sujet de la carrière criminelle réussie}

\section{Lise Lalonde et Carlo Morselli}

Volume 14, numéro 1, automne 2001

Où est la marge ?

URI : https://id.erudit.org/iderudit/1074159ar

DOI : https://doi.org/10.7202/1074159ar

Aller au sommaire du numéro

Éditeur(s)

Université du Québec à Montréal

ISSN

1180-3479 (imprimé)

1916-0976 (numérique)

Découvrir la revue

Citer ce document

Lalonde, L. \& Morselli, C. (2001). Une carrière en marge de l'autre : entretien avec un criminologue au sujet de la carrière criminelle réussie. Frontières, 14(1), 49-52. https://doi.org/10.7202/1074159ar d'utilisation que vous pouvez consulter en ligne.

https://apropos.erudit.org/fr/usagers/politique-dutilisation/ 


\section{UNE CARRIÈRE EN MARGE DE L'AUTRE Entretien avec un criminologue au sujet de la carrière criminelle réussie}

\author{
UNE ENTREVUE RÉALISÉE PAR \\ Lise Lalonde, Ph.D., \\ Sciences humaines appliquées, avec \\ Carlo Morselli, \\ professeur adjoint à l'École de criminologie \\ de l'Université de Montréal.
}

Dans l'ensemble, la population considère les figures du crime organisé comme des criminels plutôt que des hommes d'affaires ayant une carrière non conventionnelle réussie. J'ai connu Carlo Morselli alors qu'il terminait des études doctorales sur cette question. Son étude portait sur les «entrepreneurs criminels » ayant plutôt bien réussi dans leur domaine d'activité. Le fait qu'ils aient pu être incarcérés n'assombrit pas leur succès - le concept de «réussite criminelle» étant fonction des relations qu'ils ont créées au sein de leur réseau et de la fortune qu'ils ont pu en retirer.

L'un des aspects originaux de la thèse de Carlo réside dans son choix de la théorie des trous structuraux de Ron Burt (1992). Je reprends, en ses mots, la fascinante dynamique paradoxale qui confère son succès à l'entrepreneur criminel :

La théorie des trous structuraux formalise le caractère performant de l'absence de connection, absence qualifiée alors de trou dans la structure relationnelle. Par ces trous, il est possible en effet de montrer comment un individu progresse en affaires en faisant des liens de manière non redondante. Des acteurs bien connectés, dans ce sens, sont souvent des acteurs stratégiquement déconnectés. Cet état de déconnectivité permet alors de maintenir des avantages compétitifs dans leurs activités pécuniaires et dans leur carrière en général.

De plus, le concept de trou structural est utilisé pour appréhender les opportunités entrepreneuriales au sein d'un réseau qui permettent à une personne de se positionner comme courtier entre des contacts déconnectés. Par le trou, et par cette position de courtier, un individu dont le temps et l'énergie sont limités, peut agir de manière plus efficace. En effet, plus l'accès de l'individu à ces opportunités est grand, plus le niveau de déconnectivité dans le réseau (c'est-à-dire, une proportion élevée de contacts non redondants) est élevé, plus le potentiel de succès devra s'accroître pour l'individu concerné (Morselli, 2001).

Pourvu que certaines conditions soient présentes, plus certaines personnes sont en marge de l'organisation criminelle, plus elles peuvent signer une carrière criminelle réussie. Carlo avait appliqué cette théorie à deux figures bien connues du monde criminel. L'entretien qui suit s'est déroulé à distance, en anglais, et j'en ai ensuite effectué la traduction. Entrevue virtuelle faite d'échanges dynamiques avec, comme point de référence, une autre figure qui a transité dans ce monde.

$\mathrm{Au}$ fil des pages, certaines de nos prémisses seront mises en marge. La non-marge est-elle si éloignée que cela de la marge ? La frontière entre marché légitime et marché illégitime est-elle aussi définie qu'on le pense? Dans le scénario qui suivra, la frontière la plus imperméable est celle qui, au nom de la réintégration, empêche de transposer à une entreprise légitime l'expertise acquise au sein d'une entreprise criminelle, mais qui pourrait profiter à toutes les parties. Sur un autre plan, on voit émerger d'autres dimensions théoriques et épistémologiques de la question, notamment l'importance des mots, des définitions, du chercheur ou de l'intervenant et de leurs choix théoriques par rapport à la construction de la réalité.

$$
\cdots
$$

LISE LALONDE: À l'origine, Carlo, nous devions interviewer une personne qui a eu une "carrière réussie» au sein d'un grand cartel de la drogue. Depuis, son agent de probation lui a déconseillé de parler à quiconque de son vécu criminel. Pour amorcer cet entretien qui tient lieu de celui que nous avons "mis en marge», peux-tu dresser le profil de cette personne?

CARLO MORSELLI : Sa carrière m'était connue par l'entremise de la couverture médiatique de ces dernières années. J'avais aussi réalisé une série d'observations à partir de données des services de renseignements criminels et d'enquêtes. Le profil de cette 
personne s'y retrouvait. Elle était associée à des membres d'un réseau de drogue ciblé par les enquêteurs. Nous nous sommes rencontrés une seule fois, en janvier dernier, pendant trois heures, et n'avons pas tenté de nous revoir.

Dans le milieu, cette personne occupait une place unique. Elle était une participante marginale d'une entreprise déjà marginale - une femme investissant la scène de l'importation de la drogue. Voici donc ce que je savais d'elle ou que j'ai appris lors de ma rencontre avec Martha - appelons-la ainsi pour préserver son anonymat. Elle sortait de prison et y avait passé six années pour sa participation à un réseau d'importation de cocaïne. Son expérience est révélatrice par rapport aux problèmes associés au trafic de la drogue, en particulier, et au contrôle du crime organisé, en général.

Au départ, Martha fut élevée au rang de figure clé du réseau. Cela est fréquent. Il semble que l'attribution d'un statut de kingpin ou de pilier - dans ce cas-ci de queenpin - à une personne en état d'arrestation soit une stratégie courante des forces de l'ordre. Il est vrai que certains de ceux qu'on arrête peuvent être la figure clé d'un réseau de trafic de drogues. Néanmoins, lorsqu'on examine les différents dossiers et le nombre de ces «chefs » arrêtés, cette pratique d'étiquetage apparaît douteuse et facile à réfuter. Tous semblent être la figure centrale d'un réseau quelconque alors que tous, on le comprendra, ne peuvent pas être le chef. Manifestement, certains de ceux qu'on désigne piliers sont loin de dominer dans quelque réseau que ce soit. En réalité, les chefs sont peu nombreux. L'attribution de ce statut de prestige à autant de suspects nous indique que le statut de plusieurs est inapproprié. En bout de piste, cette pratique répandue ne réussit qu'à banaliser l'idée d'un doyen gérant ces activités prohibées.

Plutôt que le rôle du chef autoritaire typique ou du pilier, je préfère des termes du monde des affaires qui représentent la capacité de s'intégrer aux autres. Par le passé, la notion de « courtier » m'est apparue convenir le mieux à cet égard - et les expériences de Martha s'accordent bien avec cette vision des choses. En bref, dans un contexte commercial, le courtier se situe entre les acheteurs et les vendeurs. Les risques qu'il encourt sont relativement minimes puisque le courtier n'investit pas son propre capital financier. Il mise plutôt sur le capital financier des deux parties à la transaction qui convergent indirectement par son entremise. Cependant, si les risques qu'il prend sont faibles, la possibilité d'accroître ses profits est considérable. En effet, la position du courtier favorise la constante expansion de ses services d'intermédiaire en direction d'autres contextes de transactions entre acheteurs et vendeurs. Dans le cadre d'entreprises criminelles, cette position est cruciale puisque le courtier crée une zone tampon entre l'acheteur et le fournisseur d'un produit illégal. Ces cocontractants ne se connaissant pas, ils ne peuvent s'identifier mutuellement. Quant à lui, le courtier maintient sa distance par rapport à une activité normalement sous la surveillance des forces de l'ordre.

Martha servit de courtier entre un groupe non spécifié de Colombiens exportateurs de cocaïne et plusieurs groupes d'importateurs à Montréal. Elle n'était donc pas un pilier ou une figure centrale au dossier, mais une actrice en périphérie. Pour le dire autrement, elle était un acteur parmi d'autres dans la chaîne transmettant la cargaison du producteur au consommateur. Il est difficile de concevoir qu'une ou quelques personnes contrôlent un tel circuit. Il est plus probable que le trafic de la drogue soit comparable à un "processus de patate chaude» : le produit illicite (ou l'information sur sa localisation) est transmis aussi vite que possible d'un participant à un autre. On réduit ainsi le risque d'être détecté tout en léguant la responsabilité au prochain contact.

À des fins de contrôle officiel, il s'avère commode de diffuser l'image d'une ou deux personnes à la tête d'une vaste organisation criminelle de type cartel ou monopole. Cela permet de diriger l'effort des corps policiers vers ces cibles créées de toutes pièces. C'est le scénario officiel typiquement associé au crime organisé. Or la recherche dans ce domaine d'activités nous présente une image tout autre: celle d'une organisation sociale et d'une coordination de contextes illicites qui procèdent d'un arrangement beaucoup plus desserré et désordonné. Dans une thèse intitulée Disorganized Crime, l'économiste américain Peter Reuter (1983) démontrait que, dans les faits, les marchés du prêt usurier, du bookmaking et de la loterie clandestine - secteurs perçus comme fortement contrôlés par la mafia new-yorkaise - étaient surtout gérés par des groupuscules éphémères de coparticipants. Ceux-ci n'avaient jamais atteint de contrôle du genre exhibé par les monopoles ou les cartels. Typiquement, de tels marchés illicites étaient sous l'égide de groupes indépendants et dont la structure était moins que resserrée. Dans une autre étude, Reuter et John Haaga (1989) constataient une structure de marché similaire parmi les importateurs de drogues illégales. Une étude de Patricia Adler (1993) qui remonte au début des années 1980 allait dans le même sens: elle remarquait que sa communauté de fournisseurs de marijuana puis de cocaïne du Sud-Ouest américain correspondait à la thèse $\mathrm{du}$ crime désorganisé.

Comme on peut le constater, cette idée voulant que le crime organisé se présente sous une forme strictement pyramidale et formellement structurée a été substantiellement réfutée depuis quelques années. Pour Martha et pour plusieurs autres participants au commerce des drogues illégales, le problème est le suivant: les perceptions des autorités policières et judiciaires se fondent généralement sur cette image erronée. Et pourtant, Martha n'a jamais contrôlé aucun cartel de cocaïne. Elle entretenait de très bons liens de travail avec les Colombiens fournisseurs de cocaïne et avec les importateurs de ces produits résidant à Montréal et dans les environs. C'était un arrangement tout à fait commode et elle était bien à sa place parmi les autres.

Martha a aussi été trouvée coupable de blanchiment d'argent. Elle a abordé le sujet brièvement lorsque s'est posée la question à savoir comment elle gagnerait sa vie durant ses six années de libération conditionnelle. Comme tu le disais, son agent de probation lui a conseillé de ne pas nous rencontrer. Selon ce qu'elle a compris de cette condition partielle à sa libération, notre rencontre aurait été nuisible au processus de réhabilitation qu'on veut qu'elle poursuive. Autrement dit, sa description d'activités passées n'était pas considérée bénéfique à sa réintégration dans la communauté. Martha m'apprit donc que, pour les besoins de sa libération conditionnelle, on lui assignait un travail de réceptionniste. Je voyais bien que ce type de travail n'était ni stimulant ni significatif 
pour elle. Elle sentait qu'elle avait de beaucoup dépassé ce type d'emploi. J'étais d'accord. Voici une femme qui, pendant plusieurs années, avait cultivé des contacts avec une variété de banquiers spécialisés en investissements. Elle avait acquis une expérience enviable au plan de la négociation de sommes importantes et possédait une grande connaissance des instruments de placement de haut niveau. Cela lui avait conféré une grande aisance auprès des cadres d'établissements bancaires qu'elle voyait régulièrement lorsque les profits de sa participation au commerce de la drogue lui parvenaient. Pour celui qui penserait que la capacité d'apprendre de ce type d'entrepreneur criminel plafonne vite dans un environnement de travail légitime, j'ajouterai que Martha avait complété des études graduées bien avant de s'impliquer dans ce commerce. En somme, cette femme avait exercé sa compétence auprès des hautes sphères du domaine bancaire, était habile à coordonner les transferts internationaux de produits plutôt dispendieux et, de plus, était très instruite. En l'obligeant à être réceptionniste, on favorisait son retour au commerce illégal plutôt que l'acquisition de valeurs sociales tout autres. À mon avis, seul un contexte de travail légitime qui capitalise sur ses grandes habiletés peut la satisfaire et la valoriser comme le faisait le trafic de la drogue. Il ne s'agit pas de défendre les trafiquants de drogues illégales, mais de déplorer que tant de détenus soient limités dans leur choix d'emploi en sortant de prison.

\section{LL: La carrière de Martha n'est-elle pas en marge d'une carrière typique dans le domaine criminel?}

$\mathbf{C M}$ : Il est vrai que les personnes comme Martha sont plutôt rares et que, de ce fait, elles ne sont pas représentatives du contrevenant type. D'une part, Martha était unique en ce que peu de femmes réussissent à percer un monde d'affaires dominé par les hommes. D'autre part, elle se démarquait du fait que, dans ce milieu de transit masculin et d'envergure internationale, elle s'était taillée une position privilégiée de courtier - ce qui est loin d'être évident. De son côté, le contrevenant type ne réussit pas très bien dans ses entreprises. Notre système pénitenciaire est peuplé de contrevenants qui ont commis un ou quelques larcins, mais qui sont toujours appréhendés. C'est dire que des figures comme Martha - capables de générer des profits énormes et de préserver une position d'entremetteur les assurant de continuer à recueillir de telles sommes - représentent une minorité infime en prison ou pas. La situation se compare tout à fait au monde de l'entreprise légitime: tous n'atteignent pas les sommets du succès financier.

En général, les normes d'après lesquelles on juge le succès matériel sont établies par les pairs et par ceux qui observent la scène. Pour quiconque souhaite gagner sa vie en marge de choix plus conventionnels, le standard à atteindre est bien celui de l'entrepreneur criminel qui a réussi. Ce cas de figure établit un précédent pour ceux qui s'intéressent à ce parcours: on voudra parvenir à de tels sommets de réussite. La plupart n'y parviendront pas, mais le potentiel demeure et le but est réalisable. On pourra vouloir imiter cette expérience. C'est dire que si la carrière réussie d'un entrepreneur criminel n'est pas représentative en termes quantitatifs, elle constitue un important critère de réussite à atteindre. En milieu criminel, cette dimension de la question est aussi importante que dans le milieu conventionnel: la minorité établit les normes pour la majorité qui gravit l'échelle de la mobilité sociale. Peu parviennent à ce sommet. Cependant, aussi peu nombreux soient-ils, ils sont probablement les plus remarqués et, surtout, ils exercent une influence considérable. En ce sens, la carrière criminelle réussie est sans aucun doute atypique et, par voie de conséquence, marginale du point de vue de la carrière criminelle habituelle.
LL: À ton avis, ces entrepreneurs des milieux criminels contribuent-ils à créer une marge sociale?

CM: Je ne suis pas prêt à dire que les fournisseurs créent la marginalisation qu'on remarque chez les consommateurs de drogues illégales. Par contre, leur rôle y contribue. Fais-tu allusion au processus de dépendance dans lequel se trouvent les consommateurs par rapport à leurs fournisseurs?

LL: J'entends par là le rapport entre le rôle du fournisseur de drogues illicites, la dépendance subséquente à ces drogues, la série de désaffiliations qui s'ensuivent - de la famille, des amis habituels, du travail, par rapport à un lieu de résidence établi et autres - et la marginalisation progressive de la personne qui dépend de ces substances et de son fournisseur.

CM: Si tel est le cas, on peut établir une comparaison avec l'homme d'affaires et les contextes légitimes de l'offre et de la demande. À cet effet, au milieu des années 1970, Dwight Smith (1980) s'est penché sur la définition de crime organisé ou d'activités associées à l'entreprise illégale. La théorie introduite par Smith veut que les activités prohibées et légitimes se présentent à chacun des pôles d'un continuum. Les fournisseurs de drogues pharmaceutiques légales furent situés à l'un des pôles et les négociants et les fournisseurs de drogues illégales furent placés à l'autre. On s'aperçut alors que le passage entre la légitimité et l'illégitimité participait non pas de variables comportementales, mais de la définition morale et légale de ces activités. Cela implique que la distinction entre le distributeur de drogues légales et illégales en est une de statut prohibé par rapport à un statut criminel.

Les consommateurs dépendants de drogues illégales s'inscrivent dans le même processus de marginalisation que ceux qui dépendent de drogues légales. La marginalisation des consommateurs d'hérö̈ne et de smack ressemble à s'y méprendre aux processus observés chez les alcooliques, qui en viennent à adopter les standards d'un clochard, ou chez les personnes de classe moyenne qui, à la suite d'une dépression, s'isolent dans leur propre monde. Qui crée ce marché où règne la désolation? Les fournisseurs de narcotiques? Les consommateurs eux-mêmes? Les pressions sociales? On peut dire que pour les fournisseurs légitimes et illégitimes qui ciblent ce marché, les toxicomanes potentiels représentent des occasions d'affaires en or. Donc, si les fournisseurs peuvent jouer un rôle moins significatif en termes de l'activation du marché (et ce, bien que leur rôle au départ soit plutôt directif), il est central au plan de son maintien. Les consommateurs, pour leur part, ne sont pas de simples acteurs du système. Ils jouent un rôle quant aux choix dont ils disposent. Cela dit, lorsqu'ils en arrivent à l'état de dépendance, la question du choix ne se pose plus. Là réside la distinction essentielle entre les contextes légitimes et illégaux d'offre et de demande. La prohibition, quant à elle, ajoute la dimension du vice au scénario de la dépendance - ce qui n'est pas le cas avec la problématique de l'alcoolisme ou de la dépendance au Prozac. Et le contexte social joue un rôle dans tout ça parce que la prohibition fait apparaître un double standard: à la marginalisation issue de la dépendance se greffe une marginalisation issue de la criminalité. Je ne saurais dire lequel des contextes - légitime ou illégal - est le plus profitable en termes d'affaires. Pour le savoir, il faudrait probablement comparer les coûts et les bénéfices sur les marchés ciblés par les industries pharmaceutique et les drogues illégales.

\section{LL: Explicite, si tu le veux bien, ton rapport théorique et pratique à la carrière criminelle réussie.}

CM: Dans l'ensemble, une incursion dans le monde du crime en vaut-elle le risque? Depuis le début des années 1980, nos politiques en matière de contrôle et de sanction du crime se sont appuyées sur la théorie de la dissuasion et sur les risques associés 
à la participation au commerce criminel. Lidée est la suivante : si l'on augmente les coûts associés à un crime, les contrevenants en puissance évalueront la situation et décideront, en toute logique, de ne pas exécuter le projet. Il s'agit de la théorie du choix rationnel : les décisions sont prises suivant une évaluation des coûts et des bénéfices. L'équation est simple et, à la limite, simpliste.

Je ne suis pas un ardent disciple de la théorie de la dissuasion ou du choix rationnel. Par contre, une dimension de l'équation m'est apparue intéressante: en examinant le processus de décision d'une personne qui songe à s'impliquer dans un crime, l'aspect coûts avait préséance. L'aspect bénéfices était relativement peu exploré. Pierre Tremblay et moi (Tremblay et Morselli, 2000), nous nous sommes intéressés à ce vide de la théorie du choix rationnel : les gains obtenus du crime. Elle devint la question pour nous et pour d'autres puisqu'un collège invisible se développe par rapport à la problématique des gains monétaires générés par le crime. Une étude de Bill McCarthy et John Hagan (2001) ainsi que le mémoire d'un étudiant de maîtrise (Robitaille, 2001) ont porté sur le concept de compétence criminelle. C'est dire que la question du succès dans le domaine criminel - auparavant perçu comme étant peu signifiant, donc ignoré par les milieux universitaires et politiques - prend de l'envergure comme champ d'intérêt.

Pierre et moi avons poursuivi notre étude sur le plan empirique. Un groupe de chercheurs a visité plusieurs prisons du sud du Québec afin d'interviewer les détenus et leur demander quels avaient été les revenus obtenus de leurs crimes. Puisque notre approche conçoit le crime comme une alternative aux manières conventionnelles de gagner sa vie - et qu'elle est donc exempte de jugements conventionnels -, les détenus se sont montrés plutôt réceptifs. Habituellement, dans ce domaine, on ne demande pas à un détenu combien les activités menant à sa détention ont rapporté. Cependant, nous nous intéressions aux résultats financiers - ce que les Anglais appellent the bottom line. "Tu commets des crimes pour de l'argent? Alors voyons à quel point tu $\mathrm{y}$ as réussi!» On ne se surprendra pas du fait que nos prisons soient remplies de gens sans grand talent en la matière : peu d'entre eux récoltent beaucoup d'argent de leurs crimes. En entrevue, j'ai aussi noté que ceux qui réussissaient le moins bien étaient les plus susceptibles d'adopter le langage et le protocole des services correctionnels. Certains de ceux avec des gains faibles se refusaient à concevoir le crime en termes de gains - ils n'y voyaient que les pertes. Ceux avec des gains élevés étaient bien différents. Certains d'entre eux avaient mis au point des coups merveilleux qui leur permettaient de recueillir, systématiquement et à long terme, des sommes considérables. On comprend le défi de détourner ceux qui sont capables de toucher des gains élevés de l'idée que le crime est une option valable. Et ne nous leurrons pas: ça ne prend pas des gains d'un million de dollars pour rendre l'option criminelle séduisante. En démontrant que le crime peut procurer un revenu moyen de 30000 \$ à 50000 \$ par an, le tour est joué. La plupart des détenus avaient un travail légitime régulier, mais plusieurs se repliaient aussi sur un système de gains provenant de revenus de sources criminelles et légitimes. Ce type d'information ne nous serait pas accessible par le biais de l'analyse typique en termes de coûts et de bénéfices.

En changeant de paradigme, les questions de récidive et d'abandon de la carrière criminelle prennent de l'importance et les bénéfices du crime deviennent une clé pour comprendre ces événements. Cette approche peut sembler perverse, mais elle est riche et prometteuse en termes d'enquêtes futures. À plusieurs égards, cette problématique émergente n'est pas nécessairement liée aux carrières criminelles réussies. Elle est plutôt articulée au crime comme mode de vie et à l'influence de cette perspective sur les formes de réponses et de contrôles (ou de coûts) qu'on y applique. Il est encore tôt pour considérer les enjeux politiques de l'approche que nous développons. Nous serons en mesure de les préciser lorsque les analyses de l'enquête auprès des détenus auront été complétées.

\section{LL: En conclusion, comment résumerais-tu ta perspective par rapport à la carrière criminelle réussie?}

CM : Le raisonnement est le suivant. 1) Dans notre société, il est nécessaire de gagner sa vie pour survivre. 2) Le degré d'intérêt et de signification que revêtent les activités rémunératrices disponibles varient d'une personne à une autre. 3) Ceux pour qui les activités rémunératrices disponibles présentent peu d'intérêt et de signification tendent à considérer le crime comme une alternative possible pour gagner leur vie. Triompher du système, voilà l'enjeu ! 4) Dans le contexte criminel, les options varient aussi en degré. C'est une question de compétence criminelle ou de compétence tout court. Il s'ensuit que les options qui consistent à gagner sa vie dans le domaine criminel peuvent chevaucher l'ensemble des options disponibles. 5) Puisque, pour certains, le crime représente une manière de gagner sa vie, la prison est une réalité potentielle. En d'autres mots, chacun est confronté à son propre système de taxation. 6) La question de savoir si la prison (l'un des coûts rattachés au crime) a une incidence sur la persévérance dans le milieu criminel (ou sur la récidive) dépend de la faisabilité ou du degré de signification de cette option, à l'origine, pour la personne. 7) Pour plusieurs, selon sa faisabilité (et donc selon l'habileté ou la compétence de la personne), le crime peut demeurer une alternative valide et bien compréhensible comme moyen de gagner sa vie - et ce même après la prison.

En somme, notre monde exige que chacun de nous gagne sa vie et, pour la plupart, cette condition est imposée. En corollaire, il est attendu de nous que nous choisissions une source de revenus convenable. Cela étant, je comprends tout à fait ceux qui optent pour une trajectoire alternative et qui attribuent leur décision à ces contraintes sociales. Si je dois gagner ma vie, j'entends le faire à ma façon. C'est l'essentiel de cette perspective.

\section{LL: Nous avons là matière à réflexion. Merci, Carlo, d'avoir partagé ta compréhension d'un univers que nous connaissons si peu.}

\author{
Bibliographie \\ ADLER, P.A. (1993). Wheeling and Dealing: Ethnography of an \\ Upper-Level Drug Dealing and Smuggling Community, $2^{\mathrm{e}}$ édition. \\ New York, Columbia University Press.
}

BURT, R.S. (1992). Structural Hole: The Social Structure of Competition. Cambridge, Harvard University Press.

McCARTHY, B. et J. HAGAN (2001). "When crime pays: Capital, competence, and criminal success », Social Forces, n ${ }^{\circ}$ 79, p. 10351059.

MORSELLI, C. (2001). Contacts, Opportunities, and Crime: Relational Foundations of Criminal Enterprise, École de criminologie, Montréal, Université de Montréal, 244 p. et annexes.

REUTER, P. (1983). Disorganized Crime: The Economics of the Invisible Hand. Cambridge, MIT Press.

REUTER, P. et J. HAAGA (1989). The Organization of High-Level Drug Markets : An Exploratory Study. Santa Monica, Rand.

ROBITAILLE, C. (2001). Gains criminels et facteurs individuels de réussite: une ré-analyse du sondage de 1978 de la Rand Corporation. Mémoire de maîtrise, École de criminologie, Université de Montréal.

SMITH, D.C. (1980). "Paragons, pariahs, and pirates: A spectrumbased theory of enterprise ", Crime and Delinquency, n ${ }^{\circ}$ 26, p. 358386.

TREMBLAY, P. et C. MORSELLI (2000). "Patterns in criminal achievement: Wilson and Abrahamse revisited », Criminology, $\mathrm{n}^{\circ} 38$, p. 633-659. 